\title{
Queixa vocal e análise perceptivo-auditiva de pacientes com doença de Parkinson
}

\author{
Gisele Gasparini* \\ Giovana Diaféria** \\ Mara Behlau***
}

\begin{abstract}
Resumo
A doença de Parkinson (DP) é uma alteração degenerativa do sistema nervoso central caracterizada por perda progressiva dos neurônios da substância negra. Além dos sintomas primários (rigidez, tremor e bradicinesia), alterações de voz, fala e deglutição também podem estar presentes na DP. O objetivo do presente estudo foi realizar o levantamento da queixa vocal, análise perceptivo-auditiva por meio da escala GIRBAS e do ataque vocal. Participaram 118 pacientes, 74 do gênero masculino e 44 do feminino, que buscaram atendimento na Associação Brasil-Parkinson, no Serviço de Fonoaudiologia, em 2001. Voz fraca foi a queixa mais encontrada, as alterações vocais de grau moderado e ataque vocal isocrônico. Tais achados sugerem que os pacientes parkinsonianos que procuram tratamento apresentam disfonia moderada, caracterizada principalmente por astenia e instabilidade. A utilização de diferentes tarefas de fala é necessária para uma melhor caracterização da alteração vocal.
\end{abstract}

Palavras-chave: Voz. Doença de Parkinson. Terapia. Avaliação.

\section{INTRODUÇÃO}

A doença de Parkinson é caracterizada clinicamente por tremor de repouso, rigidez, bradicinesia, distúrbio dos reflexos posturais, fenômeno da roda denteada, além de alteraçôes de voz e fala.

Aproximadamente $75 \%$ dos indivíduos com DP apresentam problemas de voz e fala, tais como: intensidade reduzida, modulação restrita, voz monótona, alteração de entonação, frases curtas, velocidade de fala alterada, jatos de fala e articulação imprecisa (RAMIG, 1995; RAMIG et al. 2001).
Todo o mecanismo fonatório na DP encontra-se afetado, uma vez que funçôes neuromusculares são necessárias para produção de fala inteligível. Fraqueza, alteração de mobilidade, atrofia e fadiga muscular produzem, certamente, desvios do início e do término da fonação (GALLENA et al., 2001).

A literatura relata que rouquidão, soprosidade, aspereza e tremor são características perceptuais da voz nos pacientes com DP (LOGEMANN et al., 1978; BAUMGARTNER; SAPIR; RAMIG, 2001; PASSOS et al., 2001). Alguns autores ainda relatam que a rouquidão e a soprosidade são os desvios mais significativos.

\footnotetext{
* Especialista em Voz, vice-coordenadora do Curso de Especialização em Voz do Centro de Estudos da Voz Rua Machado Bittencourt, $36110^{\circ}$ andar cj. 1004 Vila Mariana 04044-001 São Paulo SP Brasil

** Especializanda em Voz e Motricidade Oral. Coordenadora do Setor de Fonoaudiologia da Associação Brasil-Parkinson

*** Professora do curso de Pós-graduação da UNIFESP e diretora do Centro de Estudos da Voz
} 
A escala GRBAS, com cinco parâmetros, proposta pela Japanese Society of Logopedics and Phoniatrics se tornou a escala mais amplamente utilizada para descrever os desvios da qualidade vocal (BEHLAU; YAMAGUCHI; ANDREWS, 2001). Dejonckere e colaboradores (1996) introduziram o parâmetro "I" instabilidade, que parece ser bastante importante para a avaliação de alteraçôes neurológicas. Desse modo, escolhemos a escala GIRBAS para a avaliação das características perceptuais da disfonia hipocinética da DP.

O objetivo do presente trabalho foi correlacionar a queixa vocal com os resultados da análise vocal perceptivo-auditiva e o tipo de ataque vocal mais freqüente de pacientes parkinsonianos.

\section{MATERIAIS E MÉTODOS}

Participaram do presente estudo 118 indivíduos com diagnóstico neurológico da doença de Parkinson, 74 homens e 44 mulheres, com idade entre 43 e 89 anos, idade média de 67 anos, com estágio 2 da doença, medicados e com acompanhamento neurológico. Todos os pacientes procuraram atendimento no Serviço de Fonoaudiologia da Associação Brasil-Parkinson (ABP), em 2001.

Os dados de queixa vocal foram obtidos por meio do levantamento dos protocolos de avaliação aplicados aos pacientes no seu primeiro contato com o setor. O protocolo de avaliação é realizado por meio de entrevista com perguntas dirigidas, que são respondidas tanto pelo paciente como pelo acompanhante.

Para a realização da análise perceptivo-auditiva com a escala GIRBAS foi utilizada a vogal "a" sustentada em freqüência e intensidade habituais.

O ataque vocal foi avaliado por meio de duas tarefas diferentes: vogal sustentada e fala encadeada (contagem de números de 1 a 20). $\mathrm{Na}$ contagem de números, os juízes avaliaram o início dos vocábulos "um", "oito" e "onze".

A sala utilizada para realização das gravações não era acusticamente tratada, porém não havia ruído excessivo em seu interior. As grava- çôes foram feitas por meio de um gravador digital portátil Minidisc Recorder MD-MT15(S) com um microfone Le Son condenser MP - 68 cardioid posicionado a $10 \mathrm{~cm}$ da boca em um ângulo de $45^{\circ}$.

Para a avaliação auditiva, cada amostra de fala foi apresentada, pelo menos três vezes, a sete fonoaudiólogos treinados do Centro de Estudos da Voz (CEV). Os fonoaudiólogos classificaram os ataques vocais em isocrônico, brusco e soproso, além de avaliar a qualidade vocal por meio da escala GIRBAS, de acordo com o nível de desvio percebido para cada um dos diferentes parâmetros da escala.

A Figura 1 apresenta o protocolo de avaliação da escala GIRBAS, com quatro marcas visuais

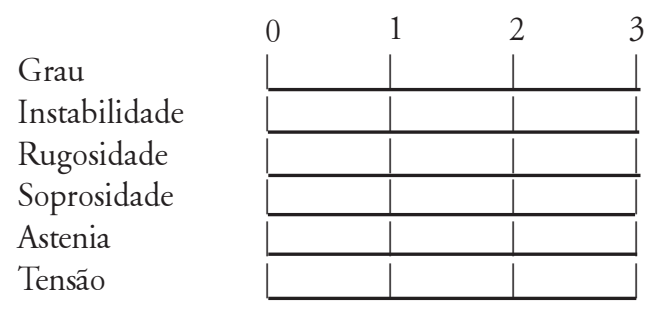

FIGURA 1 - Escala GIRBAS para análise de qualidade vocal

analógicas (sem desvio - 0 , desvio discreto - 1 , desvio moderado - 2, desvio severo - 3). O parâmetro G - grade (grau) é o desvio global vocal, o I - instability (instabilidade) é a variação anormal da voz, o $\mathrm{R}$ - roughness (rugosidade) é a impressão de irregularidade de vibração das pregas vocais, o B - breathiness (soprosidade) é a qualidade vocal relacionada à turbulência de ar audível produzida na glote devido a escape de ar, o A - asteny (astenia) é a impressão de fraqueza durante a fonação e, finalmente, o $S$ - strain (tensão) é a impressão auditiva de esforço excessivo durante a fonação.

\section{RESULTADOS}

\section{Queixa vocal}

A Tabela 1 mostra as queixas mais comuns dos indivíduos com DP. Voz fraca foi a queixa mais encontrada (45\%), seguida de dificulda- 
des para falar (24\%), "as pessoas não entende o que eu falo" (16\%), e rouquidão (11\%).

\section{TABELA 1}

Porcentagem de queixas vocais de pacientes com DP

\begin{tabular}{lc}
\hline Queixas* & $\%$ \\
\hline Voz fraca & 45 \\
Dificuldade para falar & 24 \\
As pessoas não entendem o que eu falo & 16 \\
Rouquidão & 11 \\
Sem queixa & 8 \\
Afonia & 7 \\
Gagueira & 5 \\
Não consigo falar & 4 \\
Falta de ar & 4 \\
Voz falha & 3 \\
Voz apertada & 3 \\
Fadiga vocal & 3 \\
Bolus & 3 \\
Velocidade de fala diminuída & 3 \\
Velocidade de fala rápida & 2 \\
Voz trêmula & 2 \\
*Alguns pacientes apresentam mais de uma queixa vocal.
\end{tabular}

\section{Ataque vocal}

A Figura 2 apresenta os resultados do ataque vocal na vogal sustentada. As Figuras 3, $4 \mathrm{e}$ 5 apresentam os resultados dos ataques vocais na fala encadeada. $O$ ataque vocal isocrônico foi o mais frequiente para ambas as tarefas de fala ("a" 69\%, "um" 85\%, "oito" 79\% e "onze" $77 \%)$. No entanto, houve uma certa tendência à presença de ataques vocais soprosos na contagem de números ("um" $15 \%$, "oito" $21 \%$ e "onze" 23\%) e de ataques bruscos na vocal sustentada (23\%).

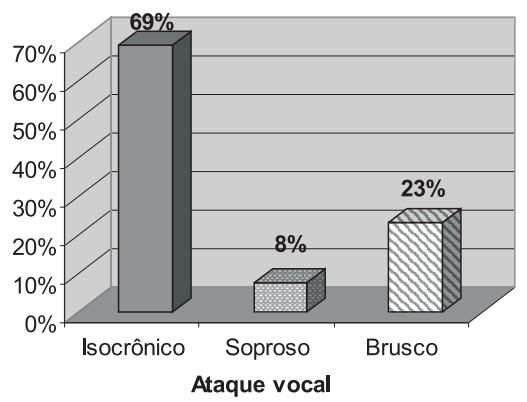

FIGURA 2 - Porcentagem de ataque vocal na vogal sustentada de pacientes com DP

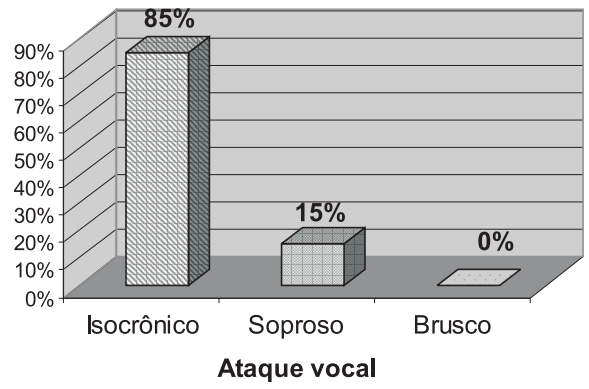

FIGURA 3 - Porcentagem de ataque vocal no vocábulo "um" durante a contagem de números em pacientes com DP

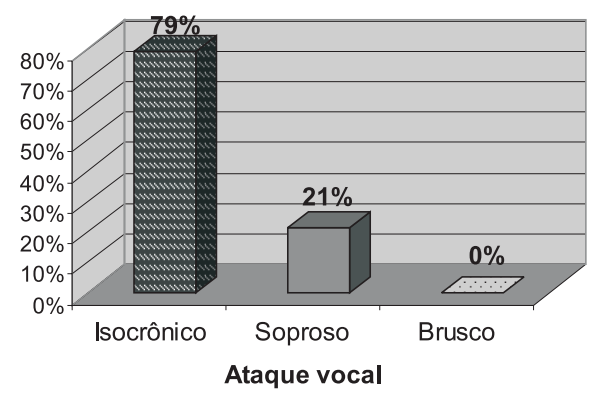

FIGURA 4 - Porcentagem de ataque vocal no vocábulo "oito" durante a contagem de números de pacientes com DP

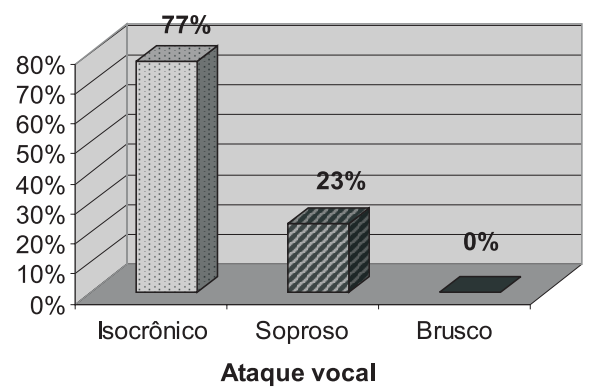

FIGURA 5 - Porcentagem de ataque vocal no vocábulo "onze" durante a contagem de números de pacientes com DP

\section{Análise perceptivo-auditiva}

A Tabela 2 apresenta os valores da média, da mediana e do desvio padrão (DP) para cada um dos parâmetros da avaliação vocal por meio da escala GIRBAS. Os resultados indicam que os indivíduos avaliados apresentam desvio vocal global em grau moderado. Os parâmetros instabilidade (média $=1,8 ; \mathrm{DP}=0,7)$ e astenia (média $=1,8 ; \mathrm{DP}=0,7)$ apresentaram maiores valores do que os demais parâmetros avaliados. 
Todos os parâmetros apresentaram desvios variando de grau leve a moderado.

\section{TABELA 2}

Valores de média e mediana dos 6 parâmetros da escala GIRBAS em 118 pacientes com DP

\begin{tabular}{lccc}
\hline & Média & Mediana & DP \\
\hline Grau & 2,0 & 2,0 & 0,7 \\
Instabilidade & 1,8 & 1,6 & 0,7 \\
Rugosidade & 1,6 & 1,6 & 0,6 \\
Soprosidade & 1,6 & 1,6 & 0,7 \\
Astenia & 1,8 & 1,6 & 0,7 \\
Tensão & 0,1 & 0 & 0,4 \\
\hline
\end{tabular}

\section{DISCUSSÃO}

O grupo de pacientes atendido no Serviço de Fonoaudiologia da ABP apresenta, como queixa mais freqüente, voz fraca, além de rouquidão, dificuldade para falar, velocidade de fala diminuída, gagueira, entre outras.

O distúrbio vocal na DP é geralmente caracterizado por intensidade reduzida, voz monótona, rouquidão e tremor. Estas características perceptuais são geralmente associadas a rigidez e hipocinesia dos músculos envolvidos na respiração, fonação e articulação (BAUMGARTNER; SAPIR; RAMIG, 2001; BAKER et al., 1997). $\mathrm{Na}$ avaliação laríngea, é comum encontrar pregas vocais arqueadas, tremor e hiperfunção supraglótica compensatória (SMITH; RAMIG, 1995). Todos esses achados confirmam a queixa vocal de voz fraca e qualidade vocal instável e astênica. O fechamento glótico incompleto impossibilita o aumento de pressão subglótica necessário para a produção de uma voz audível.

A alteração vocal nos indivíduos que buscaram tratamento na $\mathrm{ABP}$ apresentou grau de desvio global moderado. Isso se deu, provavelmente, pelo fato de os pacientes em estágio mais severo apresentarem grandes restriçôes de lo- comoção e, conseqüentemente, dificuldade para buscar atendimento, ou até mesmo pela falta de conhecimento e informaçôes sobre o tratamento. Por outro lado, podemos também levantar a hipótese de que os distúrbios vocais mais discretos geralmente não são percebidos. Os resultados da análise do ataque vocal mostraram maior freqüência de ataque vocal isocrônico em ambas as tarefas de fala. No entanto, houve uma certa tendência de ataque vocal soproso na contagem de números e de ataque vocal brusco na vogal sustentada. Supomos que a presença de ataque vocal soproso se deve ao fato de que, durante a fala encadeada, a pressão subglótica é ainda menor e, portanto, o início da emissão será mais fraco. Já a presença de ataque vocal brusco se dá, provavelmente, pela hiperfunção supraglótica compensatória.

Os resultados demonstraram que, com exceção da tensão, todos os parâmetros da escala GIRBAS mostraram-se desviados.

\section{CONCLUSÕES}

Voz fraca foi a queixa vocal mais encontrada no grupo de pacientes avaliados que buscaram atendimento no Serviço de Fonoaudiologia da Associação Brasil-Parkinson (ABPKS, SLP). Tal resultado confirma o grau de desvio discreto a moderado do parâmetro astenia obtido na análise perceptivo-auditiva. A instabilidade mostrou-se tão desviada quanto a astenia, o que dá o impacto negativo de uma fala deteriorada. Apesar de o ataque vocal isocrônico ter sido o mais freqüente em ambas as tarefas de fala utilizadas, a avaliação vocal ideal para o paciente com DP deve conter as duas tarefas, para que os resultados sejam mais confiáveis e fidedignos. Em geral, podemos concluir que tais pacientes apresentam distúrbios de voz e fala que limitam grandemente sua comunicação e, conseqüentemente, sua habilidade de inserção na sociedade. 


\title{
Vocal complaint and auditory perceptual analysis of patients with Parkinson disease
}

\begin{abstract}
Parkinson's disease (PD) is a central nervous system degenerative disease, characterized by progressive loss of the substantia nigra neurons. Besides the primary symptoms (rigidity, tremor and bradykinesia), voice, speech and swallowing disorders may be present in PD. The purpose of this study was to analyze the vocal complaints and to perform an auditory perceptual analysis (GIRBAS scale) with additional attention to glottal attack. The subjects were 118 patients, 74 male and 44 female, who have approached the "Associação Brasil-Parkinson (ABPKS)", SLP department, in 2001, for treatment. Weak voice was the main complaint, followed by moderate vocal deviations, while the glottal attack was usually regular. Those findings suggest that the PD patients who look for treatment present a moderate dysphonia characterized mainly by asteny and instability. Different speech tasks need to be used for a better characterization of the vocal problem.
\end{abstract}

Keywords: Voice. Parkinson disease. Therapy. Evaluation.

\section{REFERÊNCIAS}

BAKER, K. et al. Preliminary voice and speech analysis following fetal dopamine transplants in 5 individuals with Parkinson Disease. J Speech Lang Hear Res, v.40, p.615626, Jun. 1997.

BAUMGARTNER, C. A.; SAPIR, S.; RAMIG, T. O. Voice quality changes following phonatory-respiratory effort treatment (LSVT) versus respiratory effort treatment for individuals with Parkinson disease. J Voice, v.15, n.1, p.105-114, Mar. 2001.

BEHLAU, M.; YAMAGUCHI, H.; ANDREWS, M. Escala GRBAS em três diferentes culturas. In: CONGRESSO BRASILEIRO DE FONOAUDIOLOGIA, 9., 2001, Guarapari. Anais... CD ROM.

DEJONCKERE, P. H. et al. Differentiated perceptual evaluation of pathological voice quality: reliability and correlations with acoustic measurements. Rev Laryngol Otol Rhinol (Bord), v.117, n.3, p.219-224, 1996.

GALLENA, S. et al. Effects of levodopa on laryngeal muscle activity for voice onset and offset in Parkinson disease. J Speech Lang Hear Res, v.44, n.6, p.12841299, Dec. 2001.
LOGEMANN, J. et al. Frequency and occurrence of vocal tract dysfunctions in the speech of a large number of Parkinson patients. J Speech Hear Dis, v.43, p.47-57, 1978.

PASSOS, P. et al. Análise perceptivo-auditiva da voz de indivíduos parkinsonianos pré- e pós-terapia vocal. In: CONGRESSO BRASILEIRO DE FONOAUDIOLOGIA, 9., 2001, Guarapari. Anais... CD ROM.

RAMIG, L. O. Speech therapy for patients with Parkinson's disease. In: KOLLER, W.; PAULSON, G. (Ed.). Therapy of Parkinson's disease. $2^{\text {nd }}$ ed. New York: Basel; Hong Kong: Marcel Deker, 1995. p.539-550.

RAMIG, L. O. et al. Intensive voice treatment (LSVT) for patients with Parkinson's disease: a 2 year follow up. J Neurol Neurosurg Psychiatry, v.71, p.493-498, 2001.

SMITH, M.; RAMIG, L. Neurological disorders and the voice. In: RUBIN, J. S. et al. (Ed.). Diagnosis and treatment of voice disorders. New York; Tokio: IgakuShoin, 1995 p. 208-209.

SMITH, M. E. et al. Intensive voice treatment in Parkinson disease: laryngostroboscopic findings. J Voice, v.9, n.4, p.453-459, Dec.1995. 